\title{
COMMENTARY Cure Is Not Enough: One Slogan, Two Paradigms for Pediatric Oncology
}

\author{
Giuseppe Masera, $\mathrm{MD}^{1 *}$ Mark Chesler, $\mathrm{PhD}^{2}$ Bradley Zebrack, $\mathrm{PhD}, \mathrm{MSW}, \mathrm{MPH}^{2}$ and Giulio J. $\mathrm{D}^{\prime} \mathrm{Angio}^{2} \mathrm{MD}^{3}$
}

\section{INTRODUCTION}

Great progress has been made over the last five decades in the treatment of cancer in children. This has been due to the development of more effective combined modality treatments, and their systematic testing by consortia of institutions and cooperative clinical trials. The results have been extraordinary with gratifying increases in the survival rates for children with most of the pediatric malignant diseases.

The treatments employed were two-edged, however. Growth retardation after irradiation had been demonstrated in the early 1900's [1], but the short- and long-term complications of chemotherapy also had to be identified and defined. These iatrogenic early and late somatic effects became a matter of increasing concern. In addition, attention needed to be paid to the psychosocial and economic areas. These holistic concerns were encapsulated in the slogan, "Cure is not enough," promulgated in the 1970's [2]. The focus of pediatric oncology shifted from, "Cure at any price" when survival rates were dismal, to "Cure at least price" as effective, targeted therapies evolved. The goal became to secure long-term, complication-free survival; thus, quality of life measures became included among the major criteria of success. These aspects of care formed the first paradigm of pediatric oncology.

Some pediatric centers understood that somatic complications were not the only late effects to be avoided. They therefore embraced and applied a holistic, comprehensive approach to childhood cancer patients and their families [3]. Relevant research papers began to appear with increasing frequency in the 1990's. These documented the continuing psychosocial difficulties and post-traumatic stress symptoms experienced by many long-term survivors. Multidisciplinary teams that included family members were established. These were meant to respond to the educational, psychological, emotional, and social concerns of the child. However, as Parry and Chesler pointed out, "Most researchers have focused primarily on the negative psychosocial sequela of childhood cancer, suggesting that ... survivors manifest posttraumatic stress disorder (PTSD) [4]. The assumption that survivors of childhood cancer necessarily develop serious psychosocial difficulties or even psychopathology is not correct according to Parry and Chesler. While some patients indeed continue to suffer, most are resilient. They endure the temporary upheaval of a serious life-threatening disease, achieve or return to a normal level of psychosocial functioning, and move on to new life challenges.

By the 2000s, research had established the resilience of longterm survivors even for those who develop one or more subsequent malignancies [5]. Furthermore; recognition of post-traumatic stress gave rise to an interest in a potential corollary, post-traumatic growth (PTG). The term indicates that traumatic life events can elicit positive life changes or benefits in spite of the distressing episode [6]. Articles describing the cancer experience as a catalyst for psychosocial growth mostly appeared in the psychology literature. That literature, not often perused by medical or pediatric oncologists, includes concrete examples. Survivors often credited their cancer experience with helping them achieve greater psychological maturity, deepened personal and social relationships, and a new sense of meaning in their lives [4]. One report of 150 adolescent survivors of childhood cancer aged 11-19 years at least 1 year post-treatment is of interest. Of the 150, $127(84.7 \%)$ identified at least one positive consequence of having had cancer and $32 \%$ cited four or more benefits [7]. Other studies reported associations between older age at diagnosis and PTG or "benefitfinding [8-10]." These data suggest that having the cognitive capacity to identify one's experience as traumatic is a necessary precursor to manifesting PTG. In a study of 132 young adult survivors of high-grade osteosarcoma diagnosed before 20 years of age, those who experienced amputation (as opposed to limb surgery or no surgery) were more likely to report PTG [9]. This argues that the more severe the trauma, the higher the probability of posttraumatic growth. This observation is supported by the theoretical and empirical literature on PTG in other settings and other populations (survivors of war, terror, and sexual abuse). Individuals must first experience and acknowledge significant psychological trauma in order to be able to supervene and grow therefrom. The results of studies with regard to the association between race and gender and PTG are mixed. Some have identified a greater likelihood of reporting PTG by people of color $[8,10]$ and females [8]. Thus, some individuals who have experienced discrimination may be better able to cope with or overcome other adverse situations. The literature on alleviation of posttraumatic effects lists other helpful factors. These include supportive families and friends, time and the ability to reflect on the experience, and access to counseling. A problem-solving coping style, too, may contribute to growth. Thus, the message is not "what you have," but "what you make of it."

Pediatric oncologists have not paid particular attention to resilience or PTG, and little has been done to encourage it. Existing evidence nonetheless calls for further clinical and research attention. Meanwhile, discussion of these favorable possibilities could instill hope in survivors. Such discussions should not, however, hold out unreal or inappropriate expectations nor should parental or physician pressure be imposed. Rather, subtle, commonsense means can be employed. For example, potential patient resilience can be exemplified by photographs of sports or other celebrities who have survived cancer mounted on clinic walls.

\footnotetext{
${ }^{1}$ Department of Pediatrics, University of Milan-Bicocca, Fondazione MBBM, Monza, Italy; ${ }^{2}$ School of Social Work, University of Michigan, Ann Arbor, Michigan; ${ }^{3}$ Department of Radiation Oncology, University of Pennsylvania, Philadelphia, Pennsylvania

*Correspondence to: Giuseppe Masera, Department of Pediatrics, University of Milan-Bicocca Fondazione MBBM Via Pergolesi, 33 20900 Monza, Italy. E-mail: g.masera@hsgerardo.org
}

Received 5 December 2012; Accepted 24 January 2013 
Future investigations can proceed along two fronts. One is quantitative research through appropriately designed questionnaires. The other is qualitative and depends on the use of interviews and narratives. In both cases new measures are needed. Care must also be taken since reports of resilience and PTG can be confused. Assessments of PTG, as in some studies of post-traumatic stress studies [11], can be undertaken 6 months to 1 year after diagnosis. Evaluations can also be considered for later key time points, including the transition to off-treatment or 5-year or other long-term survival interval.

Counseling to offset PTSD and other negative psychological late-effects is certainly needed. The remarkable PTG experienced by some long-term survivors should, however, be fostered and given the prominence it certainly deserves. Awareness can be enhanced by inclusion of PTG on the agendas of relevant scientific committees, meeting programs and in long-term follow-up guidelines. These should encompass not only late complications, but also PTG as a positive late-effect.

It has become the second paradigm of pediatric oncology. Realization of its importance should strengthen the bonds between pediatric oncologists and psychologists, and thus improve clinical care and stimulate collaborative research.

\section{REFERENCES}

1. Perthes G. Über den Einfluss der Roentgen-strahlen auf epithelial Gewebe insbensondere auf das Carcinom. Archiv Für Klinische Chirurgie 1903;7:955-982.

2. D'Angio GJ. Pediatric cancer in perspective. Cure is not enough. Cancer 1975;35:866-870.

3. Masera G, Spinetta JJ, Jankovic M, et al. Guidelines for a therapeutic alliance between families and staff: A report of the SIOP Working Committee on Psychosocial Issues in Pediatric Oncology. Med Pediat Oncol 1998;3:183-186

4. Parry C, Chesler M. Thematic evidence of psychosocial thriving in survivors of childhood cancer. Qual Health Res 2005;15:1055-1073.

5. Korenjak R, Sajko MC, Jereb B. Psychosocial status of childhood cancer survivors who develop one or more secondary malignancies. Acta Medica Academica 2011;40:140-151.

6. Tedeschi RG, Calhoun LG. Trauma and transformation: Growing in the aftermath of suffering. Thousand Oaks, CA: Sage; 1995

7. Barakat L, Alderfer M, Kazak A. Posttraumatic growth in adolescent survivors of cancer and their mothers and fathers. J Pediatr Psychol 2006;31:413-419.

8. Zebrack B, Stuber M, Meeske K, et al. Perceived impact of cancer among long-term survivors of childhood cancer: A report from the Childhood Cancer Survivor Study. Psychooncology 2012;21: 630-639.

9. Yonemoto T, Kamibeppu K, Ishi T, et al. Psychosocial outcomes in long-term survivors of osteosarcoma: A Japanese single-center experience. Anticancer Res 2009:29:4287-4290.

10. Phipps S, Long A, Ogden J. Benefit-finding scale for children: Preliminary findings from a childhood cancer population. J Pediatr Psychol 2007;32:1264-1271

11. Kazak A, Boering A, Alderfer M, et al. Posttraumatic stress symptoms during treatment in parents of children with cancer. J Clin Oncol 2005;23:7405-7410. 